\title{
Analysis of medicinal value of peach
}

\author{
Hui Zhao \\ Henan University of Chinese Medicine, China \\ zhaohui814@126.com
}

Keywords: peach, chemical composition, pharmacological action, medicinal value

\begin{abstract}
Peach is a rose flower of peach or peach. The current medicine through the extraction process to prove that the peach contains peach polysaccharide, peach polyphenols, peach total flavonoids, vitamins, amino acids and trace elements compounds. Peach blossom contains compounds that can dilate blood vessels, clear the context, moisturize the skin, promote blood circulation, but also benefit the line of water, blood circulation, laxative effect, so can use peach blossom edema, limb, phlegm stagnation, Poor and so on. Peach in addition to the ornamental value, but also has a high medicinal value. In this paper, the medicinal value of the depth of the study, that the follow-up study of peach will bring a broad social and economic benefits.
\end{abstract}

\section{Introduction}

Peach blossom refers to the rosewood peach or peach flower. March each year for the open period of peach, so March for the peach harvest season, its dry, placed in a dry place, you can spare, is "spring in March, peach blossom" meaning. Peach blossom flat, bitter; heart by heart, liver, large intestine. Beneficial water, blood circulation of the effect, for edema, athlete's foot, phlegm, constipation, amenorrhea, madness, rash and other symptoms.

\section{Chemical composition}

Modern research on the chemical composition of peach contains less, the current medicine through the extraction process to prove that the peach contains peach polysaccharide, peach polyphenols, peach flavonoids, vitamins, amino acids and trace elements, is the main components of its details [1].

\subsection{Polysaccharides}

On the active elements of polysaccharides is still a hot topic, but the study of peach polysaccharide found less. The current commonly used peach polysaccharide preparation and purification methods are more mature, but there are still solvents, resource consumption and performance is not high and other disadvantages [2]. The polysaccharide composition of peach is extracted by the method of rotary evaporation, ultrasonic cleaning and ultraviolet spectroscopy. Through the continuous extraction and purification of the polysaccharide, the extraction process of the polysaccharide is improved by the response surface method.The development of modern goals [3]. In recent years, the experimental results show that peach polysaccharides and vitamin $\mathrm{C}$ superoxide anion has a strong role in the removal, so peach there is antioxidant capacity.

\subsection{Polyphenols}

Modern research in the acquisition of peach polyphenols often used to facilitate the operation, short preparation time and high extraction rate of ultrasonicassisted method, is now widely used in bio-active substance extraction [4]. Peach blossom contains peach polyphenols often used in medicinal and health functions, modern experimental studies have shown that polyphenols have a strongscavenging free radicals, liver and liver protection, anti-cancer, anti-tumor, blood lipid, 
Hardening and other biological activity [5], which makes peach widely used in food, medicine areas.

\subsection{Total flavonoids}

Flavonoids are a kind of phenolic compounds produced by photosynthesis. The biological activity of these compounds is mainly manifested in the fields of anti-oxidation, anti-tumor, hypoglycemic, anti-aging, strengthening immunity and so on. In the fields of food and medicine In the widespread application [6]. Peach blossom is rich in a variety of active functional phenolic compounds such as flavonoids, such active chemical components with dilated blood vessels, clear the context, moisturizing the skin, promote blood circulation function, which has to prevent dry skin, rough and wrinkles and other effects; The laxative active ingredient of peach is mainly related to roseosine $\mathrm{A}$ and $\mathrm{B}$.

Modern research usually uses macroporous adsorption resin and polyamide to isolate and purify the flavonoids in peach. These two separation methods have the advantages of low cost, high efficiency, no pollution and reproducibility, and are widely used in the separation of natural products purification. With the development and development of analytical instruments, the extraction process of total flavonoids from peach was also optimized, which provided some experimental basis for the study of the function and application of flavonoids in peach.

\section{Pharmacological effects}

Peach blossom bitter level, non-toxic, treatment of disease, inside and outside the salty. The pharmacological effects of peach and its function in the ancient medical books have sufficient records. "Do not record," said the peach can be in addition to water vapor, broken stone leaching, Tom Lee urine, eradication of intestinal insects; "Tang Materia Medica" will be applied to the operation of the peach, "Materia Medica" that peach can be used to promote the operation of the blood comfortable; "Lingnan herbs recorded" on its medication site to comment, said peach can be pedicable medicine, can cool blood detoxification, general acne; "outline" that , Peach can benefit from water phlegm, stagnation. The wind and madness, and its sexual off the discharge, the colostrum is very fast, can be gas treatment of people suffering from water to drink full, stagnation, the size of obstruction is not available, the benefits of no harm, but if the period is consumed Yin and blood, vitality.

Modern studies use the extraction process to demonstrate that peach blossom polysaccharides, polyphenols and total flavonoids contained in peach have complex biological activity and function [7]. These substances have the ability to dilate blood vessels, clear the veins, moisturize the skin, promote blood circulation, promote skin nutrition and Oxygen, promote the aging of the human body to promote the excretion of lipofuscin, melanin in the skin to prevent long-term accumulation, which can effectively prevent skin diseases such as chloasma, freckles, spots and other occur [8]. Peach blossom also contains protein and free state of amino acids, can accelerate the skin absorption, the prevention and treatment of skin water, dryness and aging and other issues is extremely effective [9], but also strengthen the skin's disease resistance, a serious threat to human health and Beautiful disease is good.

In recent years, the research on polyphenols and total flavonoids has been studied deeply. The results show that peach has antioxidant activity, but its antioxidant activity is different in different origin or different provenances. In addition, the peach also has a water, blood, laxative effect, so you can use peach blossom governance edema, limb, phlegm Yin stagnation, two will be unfavorable embolism.

\section{Clinical application}

Peach in addition to the ornamental value, but also has a high medicinal value, as a very important Chinese herbal medicine, often included in the ancient medical books, common side and 
indications as follows [10].

\subsection{Beriberi, lumbar kidney bladder water and phlegm}

Will be placed in the shade of the peach dry, take a big rose, And in the wine orally, with the appropriate Tongli function. Fasting use of this prescription, even when the turn can be six or seven lines, but the food is not digested and other things, the total diarrhea, if in this period that hunger physically weak, can take a little soft rice and boiled porridge.

\subsection{Dry fecal intestinal, pain and pain}

Mao Taohua one or two (wet), noodles thirty-two. On the medicine, and the surface for the ravioli full, cooked cooked, fasting food, to the afternoon, the belly, such as thunder, the current evil thing.

\subsection{Postpartum size constipation}

This side use peach, sunflower, talc, betel nut Chinese herbal medicine, each taste of the drug one or two. The drug pound fine, sifted into a loose, each time before taking the chopped green soup with two money.

\subsection{Heart and abdominal pain}

Peach sun dried at the end of the pestle. To water clothes two money dagger, children half money.

\section{Medicinal value and commonly used side}

Peach can be filled with blood, laxative, diuresis effect, commonly used to treat edema, athlete's foot, phlegm, stagnation, two will be unfavorable, amenorrhea, and so on. Symptoms, common medicinal side as follows.

\subsection{Beauty}

This side use peach, clove, fennel, frankincense, Agastache, Agaricum six herbs amount, research for the fine, add honey modulation into pills, swallow every day 7 pills, you can achieve the beauty of beauty effect. (Empress Dowager Cixi)

\subsection{Skin itching}

The peach placed in the shade after the dry ground into a fine, add honey modulation into a paste, rub in the affected area, can effectively prevent the skin in the spring itching.

\subsection{Floating swollen ascites}

The white peach will be placed in a cool place after the dry ground into a fine, each time with the amount of honey flushing to take. This can also be treated beriberi swollen, dry stool, poor urine.

\subsection{Acute jaundice infectious hepatitis}

Peach, white rattan amount, add water decoction into decoction.

\section{Therapeutic value}

With the rapid development of society, environmental and mental stress and other comprehensive factors lead to more and more people pay attention to diet health, and peach fragrance soft, with digestion, Qufeng sedative, Yang Xin Huoxue, laxative, moisturizing the skin , Beauty beauty effect, the common therapeutic side as follows [11].

\subsection{Peach Porridge}

This therapeutic side of the selection of ingredients for the peach, net trotters, japonica rice, 
ginger, green onion, and seasoning the amount. The peach baked out of the water containing it, the research into the end of peach; the rice washed clean; cut trotters flesh and bone, put it into the pot, add water, fire boil, skim the surface of the oil, Tune into a small fire stewed trotters, and so on when the bones will be removed, add japonica rice and peach at the end, continue to use a small fire simmer porridge, porridge boiled when adding seasoning and scallion mix well. Every other day, after the fractional hot after taking. The therapeutic side with Huoxue emollient, Yi Tong Tong milk, Feng muscle beauty, Huayu new function, apply to the face of the lactating women use. Postpartum taking therapeutic porridge, both through milk, eliminate body blood stasis, but also dilute the face stains and moisturizing the skin, tonic body.

\subsection{Peach blossom ham}

This recipe chooses fresh peach, cooked ham, sea rice, eggs and the right seasoning, the fresh peach picks the petals clean, cut into silk, and clean the sea with the rice to join the rice wine, steamed, chopped. Will lay the eggs into the soup, add the amount of seasoning seasoning. Wok fried, stir-fried ginger and then put into the tune of the taste of the eggs, will be fried, sprinkle with fresh peach silk, dried shrimps, ham silk, stir fry after holding the dish into both. Peach fragrance smell, ham delicious, with a spleen appetizer, raw levy and blood, beauty beauty effect.

\subsection{Peach lamb}

The diet side selection of peach, lamb, potatoes, carrots, several kinds of ingredients, with appropriate spices. Separate peach petals and wash them. Until the oil is hot under the seasoning and garlic at the end, and then fried lamb pieces, add fresh soup, rice wine, seasoning, with a small fire covered with pot simmer cook until half cooked, add potato pieces, carrot pieces, garlic, And then add the tomato sauce and tune the best taste, a little stew for a moment, sprinkle fresh peach petals, mix well. Peach aroma rich, lamb delicious, with kidney yang, beauty moisturizing effect.

\section{Outlook}

Peach blossom first appeared in the "Shen Nong's Materia Medica" in the record of its "good color" effect; "map by the Materia Medica" records peach can be "Yue Ze face, Jingrong Rong color", medicine Wang Sun Simiao in the selection of beauty side Is also highly respected peach. "Taiping Sheng Hui Fang" included in the prescription of the use of dried peach at the end of the compatibility of melon seeds at the end of the same amount of honey can be used for freckles, black spots, nevus treatment; "Ge Hong elbow back emergency" that Peach can be used for whitening skin, slim waist. Through the processing and extraction process that the peach blossom contains peach polysaccharide, peach polyphenols, peach total flavonoids can be used for anti-aging, free radicals, enhance immunity, clinically can be used to prevent depression, improve learning ability [12].

Peach as a food and medicine homologous products, which contains a wealth of vitamins, amino acids and trace elements chemical composition, has a good beauty and health effects [13]. "Ancient and modern secret garden" contains: "March 3 harvest peach, July 7 received chicken blood, two flavor and Mo face, to three days off, then the color Guanghua." Peach is often used as beauty to share. With the social and economic progress and material enrichment, peach in the field of skin care products gradually play an important role. Extracted from the peach oil essential oil components, but also has to promote blood circulation, prevention and treatment of melanin deposition, can effectively remove chloasma, freckles, dark spots [14].

Modern medicine on the extraction of peach processing technology is not perfect, the ingredients contained in the peach is still lack of understanding, the rational use of some new technologies may effectively improve the extraction and purification of the effect of the extraction of peach flowers to the direction of energy efficient development. Therefore, the subsequent research on the direction of the peach chemical composition and pharmacodynamics should be fully explored in order to its clinical application have a more fully understood, and then bring considerable social and economic 
benefits.

\section{References}

[1] Yang Qianqian, Sun Fangling, Ai Houxi, et al. Advances in chemical composition of peach [J]. Chinese Journal of Pharmacology and Toxicology, 2012,26 (03): 425.

[2] Lan Junfeng, Lai Hongfang. Ultrasonic-assisted semi-bionic extraction of peach polysaccharide [J] .Hubei Agricultural Sciences, 2014,53 (02): 401.

[3] Geng Mingjiang, Xi Rongying, Yu Chuanhua, et al. Optimization of extraction process of peach polysaccharide by response surface method [J]. Food Research and Development, 2012,33 (12): 29.

[4] YUAN Jian-mei, GENG Ming-jiang, YAN Rui-fang, et al.Study on optimization of process and antioxidation activity of peach polyphenols by ultrasonic extraction [J]. FOOD SCIENCE \& TECHNOLOGY, 2012,37 (12): 179.

[5] Yan Ruifang, Li Xiangrong, Yu Chuanhua, et al. Separation and Purification of Peach Polyphenols by Macroporous Adsorption Resin [J]. FOOD INDUSTRY SCIENCE AND TECHNOLOGY, 2012,33 (17): 22.

[6] Zeng Zhen, Liu Hougen, Hong Yanping, et al. Study on the process and antioxidant activity of total flavonoids in peach blossom by macroporous resin [J]. Journal of Jiangxi Agricultural University, 2017,39 (01): 182.

[7] GUO Cai-zhen, SHAO Fen-juan, YAN Gui-qin.Study on Extraction and Antioxidant Activity of Polysaccharides from Peach Blossom [J]. Chinese Traditional Medicine, 2009,40 (S1): 156.

[8] Chen Linlin, Wei Shanshan, Li Deshun, et al. Determination of polysaccharide content and antioxidant activity of peach in different habitats [J]. Agricultural Science and Technology, 2016, (32): 44.

[9] Xiong Lina. China's common consumption of flowers polyphenol components and biological efficacy of [D]. Zhejiang University, 2014.

[10]Ma Jianzhong, Zhang Youcheng, Xu Xiaodong, et al. [J]. Chinese Journal of Traditional Chinese Medicine, 2013,28 (07): 1020.

[11]Zhou Ling. Peach's medicinal diet [J]. Shandong Food Science and Technology, 2004, (11): 24.

[12] Li Wanli, Du Zhimin, Geng Mingjiang, et al. Effects of peach polyphenols on monoamine neurotransmitters in hippocampus of depressed mice and its intervention [J]. Chinese Journal of Public Health, 2013,29 (03): 371.

[13] Ma Qian. Peach polyphenols on AD model rats related to protein and learning behavior [D]. Xinxiang Medical College, 2015.

[14]Gao Mu Youzi. Chinese medicine beauty skin care side of the drug characteristics and prescription law [D]. Nanjing University of Traditional Chinese Medicine, 2009. 